\title{
Laser-Enhanced Ionization Detection of Pb in Seawater by Flow Injection Analysis with On-Line Preconcentration and Separation
}

\author{
Ching-Bin Ke and King-Chuen Lin* \\ Department of Chemistry, National Taiwan University, and Institute of Atomic and Molecular Sciences, Academia Sinica, \\ Taipei 106, Taiwan, Republic of China
}

The flame laser-enhanced ionization (LEI) technique is coupled with the flow injection analysis system to measure the trace lead amounts in aqueous solution and in seawater. The flow injection (FI) manifold is incorporated with a microcolumn packed with a $\mathrm{C}_{18}$ bonded silica. The chelating agent DDPA is used to form the Pb-DDPA complex, which may be sorbed in the microcolumn and then eluted with methanol. The preconcentrated $\mathrm{Pb}$ is then detected by the LEI technique with either single-step or two-step excitation. At 5- and $15-\mathrm{mL}$ volume-fixed sample loading, the detection limits of 0.011 and 0.0033 $\mathrm{ng} / \mathrm{mL}$ (11 and $3.3 \mathrm{ppt}$ ) and enrichment factors of 16 and 48 are achieved, respectively, using a two-step FI-LEI. The sensitivity of the current system proves to be better by at least 1 order of magnitude than that of conventional LEI method. The FI-LEI also increases the tolerance of matrix interference. The LEI signal is slightly reduced to $80 \%$ intensity as $10000 \mu \mathrm{g} / \mathrm{mL}$ (ppm) $\mathrm{Na}$ and $\mathrm{K}$ matrixes are mixed in the lead solution. The resistance to the alkali matrixes is enhanced $\sim 4$ times that reported previously using a similar water-immersed probe as a LEI collector. Finally, the FI-LEI is for the first time applied to detect the $\mathrm{Pb}$ content in seawater, achieving a result of 0.0112 $\pm 0.0006 \mathrm{ng} / \mathrm{mL}$ (ppb) consistent with the certified value of $0.013 \pm 0.005 \mathrm{ng} / \mathrm{mL}(\mathrm{ppb})$.

Laser-enhanced ionization (LEI) spectrometry in flames has been successfully developed for decades, capable of detecting trace metal elements at the subnanogram per milliliter level in aqueous solution with extremely high sensitivity and selectivity. ${ }^{1-10}$

\footnotetext{
* Corresponding author: (fax) 886-2-23621483; (e-mail) kclin@ hp9k720.iams.sinica.edu.tw.

(1) Travis, J. C.; Turk, G. C.; Green, R. B. Anal. Chem. 1982, 54, 1006A1018A.

(2) Omenetto, N.; Berthoud, Th.; Cavalli, P.; Rossi, G. Anal. Chem. 1985, 57, $1256-1260$.

(3) Axner, O.; Lindgren, I.; M agnusson, I.; Rubinsztein-Dunlop, H. Anal. Chem. 1985, 57, 773-776.

(4) Turk, G. C.; Yu, L.; Koirtyohann, S. R. Spectrochim. Acta 1994, 49B, 15371543.

(5) Wang, S. C.; Lin, K. C. Anal. Chem. 1994, 66, 2180-2186.

(6) Riter, K. L.; Clevenger, W. L.; M ordoh, L. S.; Smith, B. W.; M atveev, O. I.; Winefordner, J. D. J. Anal. At. Spectrom. 1996, 11, 393-399.

(7) Smith, B. W.; Petrucci, G. A.; Badini, R. G.; Winefordner, J. D. Anal. Chem. 1993, 65, 118-122.

(8) Turk, G. C. Anal. Chem. 1981, 53, 1187-1190.
}

10.1021/ac980970t CCC: $\$ 18.00$ C 1999 American Chemical Society Published on Web 03/06/1999
The LEI detection takes advantage of a relatively large solid angle of signal collection, a near-unity quantum efficiency, and the minimization of optical interference. These features make this technique as comparable as any optical spectrometers applied in the trace analysis. However, it suffers from electrical interference contributed from the flame components or the easily ionized matrix species introduced. ${ }^{11-14}$ Such interference may prevent the electric field applied from reaching the radiation-interactive regime, causing reduction of the sensitivity. Various methods have thus been explored to eliminate this effect. Among them are, for instance, increase of voltage applied to the electrodes, 11,14,15 employment of pulsed voltage-biased collectors, ${ }^{12,16}$ modification of electrode configuration, 8,11,14,15 pretreatment with a chromatographic method, ${ }^{17,18}$ and acidification of the sample solution. ${ }^{19}$

Recently, we developed an alternative to reduce the electrical interference by interfacing a flow injection (FI) system to the LEI apparatus. ${ }^{5,20}$ With the designed FI-LEI, a water plug injected into the sample carrier stream may cause a concentration gradient of both sample analyte and matrix species along the passage to the detector. It is this dispersion behavior that substantially reduces the interference. The FI-LEI provides another advantage to automatically search for the optimal conditions at which the LEI signal may be enhanced to the maximum extent.5,20

The FI methods, with limited sample handling, high sample throughput, rapid sampling frequency, high reproducibility, low sample consumption, and low contamination risk, prove to be superior to their batch counterparts. ${ }^{21,22}$ In addition, the $\mathrm{FI}$ system may incorporate an in-line chromatographic-like kit to separate

(9) Barker, P.; Rubinsztein-Dunlop, H. Spectrochim. Acta 1997, 52B, 459-469. (10) Butcher, D. J. In Lasers in Analytical Atomic Spectroscopy; Sneddon, J., Thiem, T. L., Lee, Y., III, Eds.; VCH: New York, 1997; Chapter 6.

(11) Turk, G. C.; Travis, J. C.; DeVoe, J. R.; O'Haver, T. C. Anal. Chem. 1979, $51,1890-1896$.

(12) Turk, G. C. Anal. Chem. 1992, 64, 1836-1839.

(13) Schenck, P. K.; Travis, J. C.; Turk, G. C.; O'Haver, T. C. J. Phys. Chem. 1981, 85, 2547-2557.

(14) Green, R. B.; Havrilla, G. J.; Trask, T. O. Appl. Spectrosc. 1980, 34, 561568.

(15) Havrilla, G. J.; Green, R. B. Anal. Chem. 1980, 52, 2376-2383.

(16) Vippoldt, M. A.; Green, R. B. Anal. Chem. 1983, 55, 554-557.

(17) Havrilla, G. J.; Carter, C. C. Appl. Opt. 1987, 26, 3510-3515.

(18) Epler, K. S.; O'Haver, T. C.; Turk, G. C. J. Anal. At. Spectrom. 1994, 9, 79-82.

(19) Trask, T. O.; Green, R. B. Anal. Chem. 1981, 53, 320-324.

(20) Wang, S. C.; Lin, K. C. Analyst 1995, 120, 2593-2599.

(21) Ruzicka, J.; Hansen, E. H. Flow Injection Analysis, 2nd ed.; Wiley: New York, 1988; Chapters 1-4. 
the analyte of interest from interfering matrixes and to concentrate its content for increasing detection sensitivity. ${ }^{22}$ Taking advantage of these merits, flow injection analysis (FIA) coupled with various detection methods has been successfully adopted to determine the quantities of some metals in biological and environmental materials at nanomolar to picomolar levels. Among these detectors, the flame atomic absorption spectrometer (FAAS) is mostly employed with $\mathrm{FI}$, others being spectrophotometry, electrothermal method, conductimetry, fluorescence, and ICPM S.22 However, the laser-based spectrometers have seldom been employed. As a detector, the LEI is anticipated to be more sensitive than FAAS in trace analysis, although both techniques share the same way for sample introduction. In this work, we therefore attempt to couple LEI with the FI system to determine the trace lead content in aqueous solution and in seawater.

Lead contained in seawater has been determined by various methods. Fang et al. achieved detection limits of $0.5-0.8 \mathrm{ng} / \mathrm{mL}$, using a dual-column on-line preconcentration FI-AAS system with different chelating ion exchangers. ${ }^{23}$ Rodriguez et al. obtained detection limits of 10.0 and $4.0 \mathrm{ng} / \mathrm{mL}$ and enrichment factors of 37 and 100 at 5-and 30-mL lead sample loading, respectively, using $\mathrm{FI}$ on-line ion-exchange preconcentration and FAAS detection. ${ }^{24}$ By applying buffer-masking solution to the ion exchangers in $\mathrm{FI}$ AAS for suppressing the matrix interference, Bysouth and Tyson showed the enhancement of lead recoveries in the tap water ${ }^{25}$ By means of a selective reaction with porphyrin in a FI manifold followed by spectrophotometric detection, Schneider and Hornig achieved lead recoveries from tap water up to $98-109 \%$ with a detection limit of $10 \mathrm{ng} / \mathrm{mL}$ when Fe was present in the sample matrix and $4.2 \mathrm{ng} / \mathrm{mL}$ when it was not. ${ }^{26}$ Fang et al. obtained a detection limit of $3 \mathrm{ng} / \mathrm{mL}$ by using a sorbent extraction AAS system. ${ }^{27}$ The sorbent extraction column was packed with $C_{18}$ bonded silica sorbent, in which the lead complex was sorbed and eluted with ethanol or methanol. FI with sorbent extraction preconcentration has also been successfully adapted to an electrothermal atomic absorption spectrometer (ETAAS) system. For instance, with the FI-ETAAS technique, a Pb detection limit of $0.003 \mathrm{ng} / \mathrm{mL}$ in seawater was achieved without affecting the normal sample throughput and sampling frequency. ${ }^{28}$ For adapting the on-line coupling of the knotted reactor with ETAAS, Sperling et al. introduced air flow in the FI manifold to reduce the eluate volume and minimize the dispersion effect.29 The resulting enhancement factor of 142 and $\mathrm{Pb}$ detection limit of $0.0022 \mathrm{ng} /$ $\mathrm{mL}$ was obtained at $6.8 \mathrm{~mL} / \mathrm{min}$ sample loading rate and $60-\mathrm{s}$ preconcentration. Among the above detection methods, it was found that the ETAAS detector may lower the limit of $\mathrm{Pb}$ detection in aqueous solution or seawater by $\sim 2$ orders of magnitude.

In this work, we have coupled the FI manifold with LEI detection. Taking advantage of the merits of FI separation and

(22) Fang, Z. L. Flow Injection Separation and Preconcentration; VCH: New York, 1993; Chapters 1-7.

(23) Fang, Z. L.; Ruzicka, J.; Hansen, E. H. Anal. Chim. Acta 1984, 164, 23-39.

(24) Rodriguez, D.; Fernandez, P.; Perez-Conde, C.; Gutierrez, A.; Camara, C. Fresenius J. Anal. Chem. 1994, 349, 442-446.

(25) Bysouth, S. R.; Tyson, J. F. Analyst 1990, 115, 571-574.

(26) Schneider, J. A.; Hornig, J. F. Analyst 1993, 118, 933-936.

(27) Fang, Z. L.; Guo, T. Z.; Welz, B. Talanta 1991, 38, 613-620.

(28) Fang, Z. L.; Sperling, M.; Welz, B. J. Anal. At. Spectrom. 1990, 5, 639646.

(29) Sperling, M.; Yan, X. P.; Welz, B. Spectrochim. Acta 1996, 51B, 18911908. preconcentration and of extreme sensitivity and selectivity for the LEI detector, we are the first to apply the FI-LEI system to detect the lead content in aqueous solution. $\mathrm{FI}$ is incorporated with a microcolumn packed with a $\mathrm{C}_{18}$ bonded silica. The chelating agent DDPA is used to form the Pb-DDPA complex, which may be sorbed in the microcolumn and then eluted with methanol. The preconcentrated $\mathrm{Pb}$ is then detected by the LEI technique with either single-step or two-step excitation. Using two-step LEI detection, the enrichment factor reaches 16- and 48-fold with detection limits of 0.011 and $0.0033 \mathrm{ng} / \mathrm{mL}$, respectively, at 5- and 15-mL volume-fixed sample loading. Here the enrichment factor is evaluated as the ratio between the slopes of the calibration curves obtained with and without a preconcentration procedure.22 The sensitivity of the current method is comparable to that obtained by ETAAS. The interference tolerance of $\mathrm{K}$ and $\mathrm{Na}$ sample matrixes can also be increased to 10000 ppm, $\sim 4$ times that reported previously. The $\mathrm{Pb}$ content in seawater is finally examined and the result is in agreement with the certified value.

\section{EXPERIMENTAL SETUP}

LEI Apparatus. The basic LEI apparatus contains three parts: flame burner assembly, metal electrode as a collector of charged particles, and laser radiation sources.

Flame System. As described elsewhere, ${ }^{30,31}$ a commercial burner assembly (Perkin-Elmer) with a $100 \mathrm{~mm} \times 0.5 \mathrm{~mm}$ slot burner head coupled with an interlocked gas control system was employed. The fuel $\mathrm{C}_{2} \mathrm{H}_{2}$ and air were regulated at flow rates of 0.5 and $12.5 \mathrm{~L} / \mathrm{min}$, respectively, and were premixed prior to reaching the burner head. The corresponding flame temperature was measured as $\sim 2500 \mathrm{~K} .32,33$

Laser Radiation. T wo tunable dye lasers (Spectra-Physics PD L$2 \mathrm{~A}$ and PDL3), each pumped by individual $\mathrm{Nd:YAG}$ lasers (Spectra-Physics, DCR-2A and GCR3) in a second harmonic generator, were used as radiation sources. One dye laser with rhodamine 590 emitted at $567 \mathrm{~nm}$, while the other laser with rhodamine 640 , dissolved in a $0.1 \% \mathrm{NaOH}$ methanol solution, emitted at $600.2 \mathrm{~nm}$. The output frequency of the former laser was then doubled through a KDP crystal emitting at $283.3 \mathrm{~nm}$ for excitation of the $\mathrm{Pb}$ atom in the ${ }^{3} \mathrm{P}_{0}-{ }^{3} \mathrm{P}_{1}$ transition. The second dye laser at $600.2 \mathrm{~nm}$ was simultaneously used to excite $\mathrm{Pb}$ from ${ }^{3} \mathrm{P}_{1}$ to ${ }^{3} \mathrm{D}_{2}$. Two beams unfocused were propagated in opposite directions along the flame axis, each through a pinhole of 3.04.5- $\mathrm{mm}^{2}$ cross section, and overlapped spatially and temporally inside the flame, $7 \mathrm{~mm}$ above the burner head. In this work, we employed two types of LEI schemes for lead atom detection. For the two-step LEI scheme, the lead atoms in the flame were stepwise excited, as described above, and then ionized collisionally. In contrast, in the detection with the single-step LEI scheme, only one dye laser at $283.3 \mathrm{~nm}$ was used. The output energies for 283.3 and $600.2 \mathrm{~nm}$ were 50 and $700 \mu$ ). The energy of the first dye laser was kept small to minimize ionization interference of background species from the burned organic solvent.

Electrodes. The detailed description for the electrode configuration may be found elsewhere. ${ }^{8,10}$ B riefly, a water-cooled cylinder

(30) Su, K. D.; Lin, K. C. Appl. Spectrosc. 1994, 48, 241-247.

(31) Su, K. D.; Chen, C. Y.; Lin, K. C.; Luh, W. T. Appl. Spectrosc. 1991, 41, $1340-1343$

(32) Ke, C. B.; Lin, K. C. Appl. Spectrosc. 1998, 52, 187-194.

(33) Su, K. D.; Lin, K. C.; Luh, W. T. Appl. Spectrosc. 1992, 42, 1370-1375. 


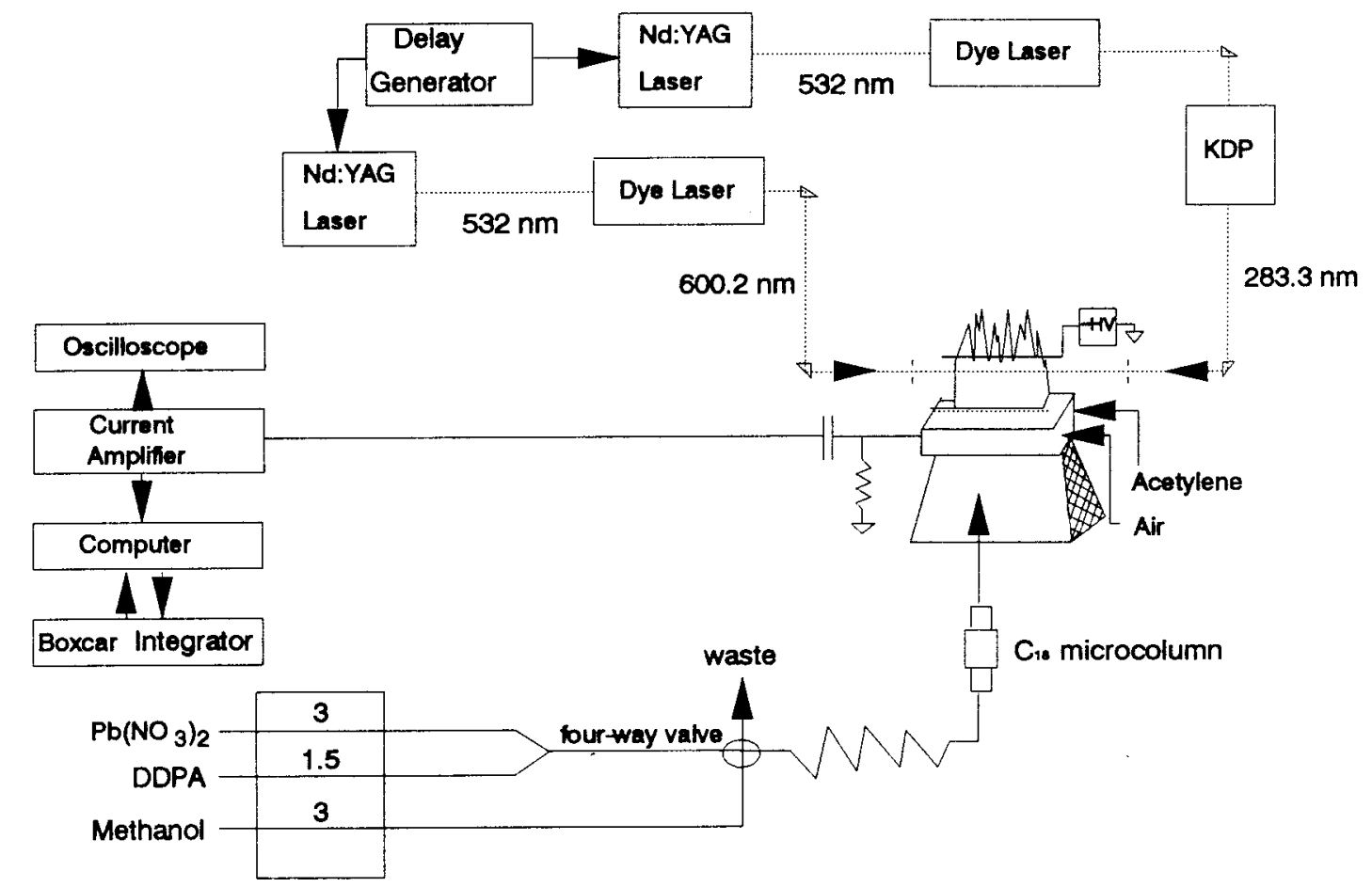

Figure 1. Schematic diagram of two-step LEI detection in combination with a FI on-line sorbent extraction system.

electrode was biased at $-1000 \mathrm{~V}$ and suspended $1.5-2.0 \mathrm{~cm}$ above the burner head. The electrode was made of $1 / 4$-in.-o.d. stainless steel tubing, through which the water was immersed. The burner served as the other electrode, from which the ion current was collected and amplified with a current-to-voltage converter (Keithley, model 428) and then fed into a boxcar integrator (PAR, models 4402, 4420, and 4422) for improved signal-to-noise ratio. The sampling rate of the boxcar integrator was fixed at 10 shots per point. The LEI signal was displayed on an oscilloscope or stored in a PC for data treatment.

Reagents. The chemicals $\mathrm{Pb}\left(\mathrm{NO}_{3}\right)_{2}, \mathrm{KCl}, \mathrm{NaCl}, \mathrm{HNO}_{3}, \mathrm{NH}_{3-}$ (aq), citric acid, and DDPA were reagent grade. The methanol solvent was spectroscopy grade. The chelating agent DDPA (ammonia diethyl dithiophosphate; 95\% Aldrich) was prepared at a concentration of $0.2 \%(\mathrm{w} / \mathrm{v})$ and buffered to a $\mathrm{pH}$ of 1 with nitric acid. The citric acid, as a masking agent to reduce the matrix interference from $\mathrm{Mn}^{2+}$ and $\mathrm{Fe}^{3+}$, was made up of a final concentration of $0.1 \mathrm{M}$ and mixed in the DDPA solution. The standard solution of $100 \mu \mathrm{g} / \mathrm{mL}$ (ppm) Pb was prepared from $\mathrm{Pb}$ $\left(\mathrm{NO}_{3}\right)_{2}$. Appropriate concentrations of $\mathrm{Pb}$ solutions were diluted for use in the work.

FI-LE I Apparatus. A schematic diagram of the flow injection manifold coupled with the LEI detector is depicted in Figure 1. The FI consists of a four-channel peristaltic pump (Ismatec), a four-port low-pressure Teflon injection valve, and a microcolumn, which was packed with bonded silica containing octadecyl functional group $\left(\mathrm{C}_{18}\right)$ as sorbent. The pump tubing of $\mathrm{FI}$ used for the carrier streams were Tygon tubings. To ensure that the flow rates were maintained constant, we replaced the pump tubings frequently, especially for the one carrying the methanol solvent. The PTFE reaction coil $(0.76 \mathrm{~mm}$ i.d. and $50 \mathrm{~cm}$ long) was knotted to minimize the dispersion of carrier streams and facilitate the chelating reaction.
In the preconcentration step, a volume-fixed $5-15 \mathrm{~mL}$ of sample containing different concentrations of $\mathrm{Pb}$ (II) buffered at an appropriate $\mathrm{pH}$ was continuously pumped into the system and mixed thoroughly with a DDPA solution. The Pb-DDPA chelating complex was adsorbed on the $\mathrm{C}_{18}$ bonded silica gel microcolumn. In the elution step, the four-port injection valve was switched to allow for injection of methanol solvent passing through the microcolumn to elute the adsorbed complex and sweep the $\mathrm{Pb}$ to the LEI detector. If the complex was incorporated with condensed alkali matrix, then deionized water was injected to rinse the residual matrix before methanol was used.

\section{RESULTS AND DISCUSSION}

FI Condition Optimization. The FI conditions are optimized in an attempt to obtain completion of chemical reaction in the coil, sufficient adsorption of the resultant chelating complex on the microcolumn, limited dispersion in the eluting process, and a large enrichment factor for the trace lead element. Under these optimized conditions, we then examine a series of effects or behavior exhibited by matrix interference, enrichment factor, and detection limit using single-step and two-step LEI detection and finally determine the $\mathrm{Pb}$ content in seawater.

Figure 2a shows the LEI signal intensity as a function of the DDPA concentration $(w / v)$, while the flow rates are fixed at 3 $\mathrm{mL} / \mathrm{min}$ for both $\mathrm{Pb}$ sample and methanol eluent and $1.5 \mathrm{~mL}$ / min for the DDPA reagent. The LEI intensity reaches a plateau when the DDPA concentration is increased to $>0.1 \%$ and the $0.2 \%$ DDPA is thus adopted in this work. Next, we examine the $\mathrm{pH}$ dependence of the Pb LEI signal. The $\mathrm{pH}$ values of the DDPA aqueous solutions are buffered ranging from 1 to 9 by adding either $\mathrm{NH}_{3}$ or $\mathrm{HNO}_{3}$ aqueous solution. The result is shown in Figure $2 \mathrm{~b}$, indicating that the acidified solution is favored. We therefore made up the DDPA solution buffered at $\mathrm{pH} 1$ throughout 

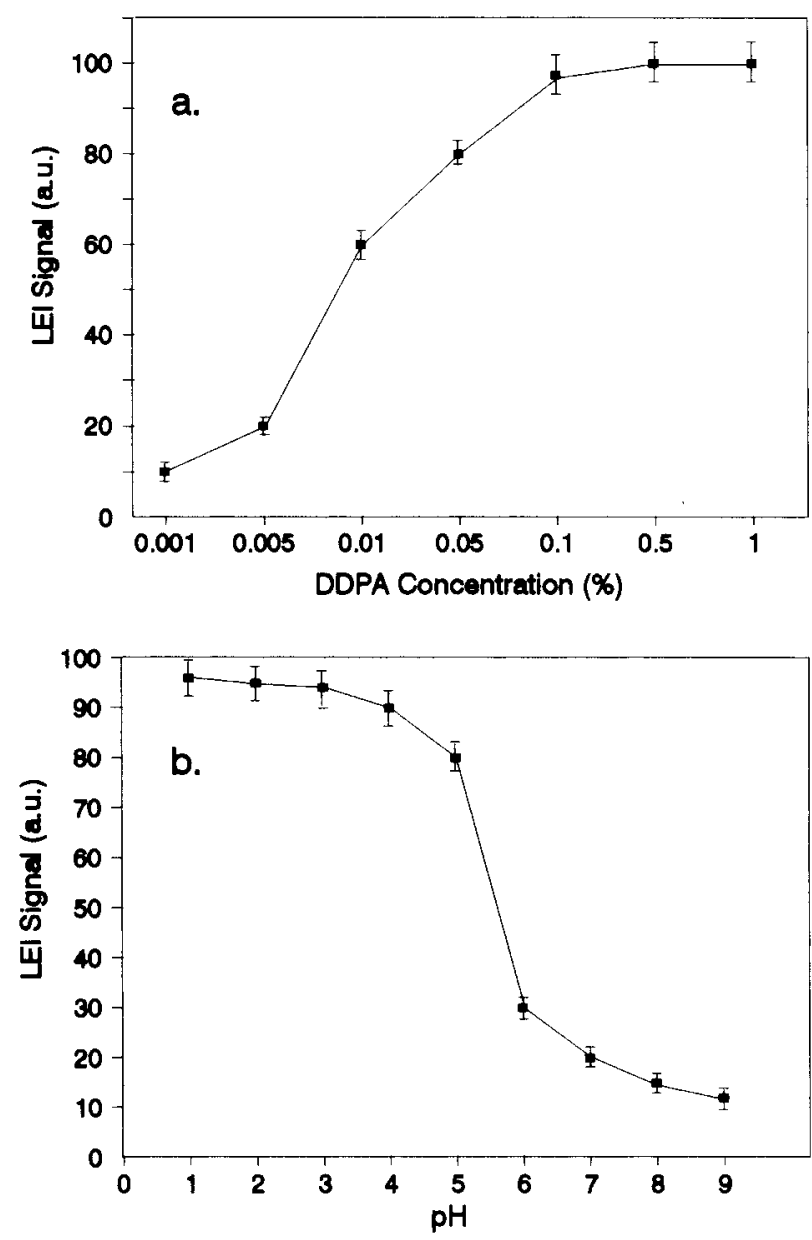

Figure 2. (a) One-step LEl signal as a function of DDPA concentration, given the flow rates of DDPA, lead sample, and methanol at $1.5,3$, and $3 \mathrm{~mL} / \mathrm{min}$. (b) One-step LEI signal as a function of $\mathrm{pH}$ value of DDPA solution with the same flow rate conditions as in (a).

the work. In order for optimization of the flow rates, $5 \mathrm{~mL}$ of 1 $\mu \mathrm{g} / \mathrm{mL}(\mathrm{ppm}) \mathrm{Pb}$ sample solution is loaded in the FI manifold and $0.2 \%$ DDPA solution at $\mathrm{pH} 1$ is continuously pumped through the passage before the methanol channel is switched on. We then successively change the flow rates of three aqueous solutions of DDPA, sample analyte, and methanol eluent. The flow rate dependence of LEI signals is shown in Figure 3. The optimized conditions are set at 3,3 , and $1.5 \mathrm{~mL} / \mathrm{min}$ for $\mathrm{Pb}$ analyte, methanol, and DDPA, respectively. Under these conditions, to complete analysis of a 5-mL sample takes $\sim 3.1 \mathrm{~min}$.

Lead Determination by FI-LEI. Lead is a cumulative toxic element to the body. Its content in natural water is normally less than $20 \mathrm{ng} / \mathrm{mL}$ (ppb). Atomic absorption spectroscopy coupled with FI separation and a preconcentration device, such as FI-FAAS, is popularly applied to the analysis of aqueous lead solutions. In this work, we first employ LEI as a detector of FIA. To find the FI-LEI capability, we compare the sensitivity obtained between the current method and its batch counterpart. Figure 4 shows the results of $\mathrm{Pb}$ sample measurement using single-step LEI detection with and without a preconcentration procedure. Each LEI intensity corresponds to the average of three replicates. The $\mathrm{Pb}$ sample solutions were prepared in the range from 25 to 100 $\mathrm{ng} / \mathrm{mL}$ (ppb); $50 \mu \mathrm{g} / \mathrm{mL}$ (ppm) Na species was added as matrix in each solution. As shown in the figure, a one-step LEI technique
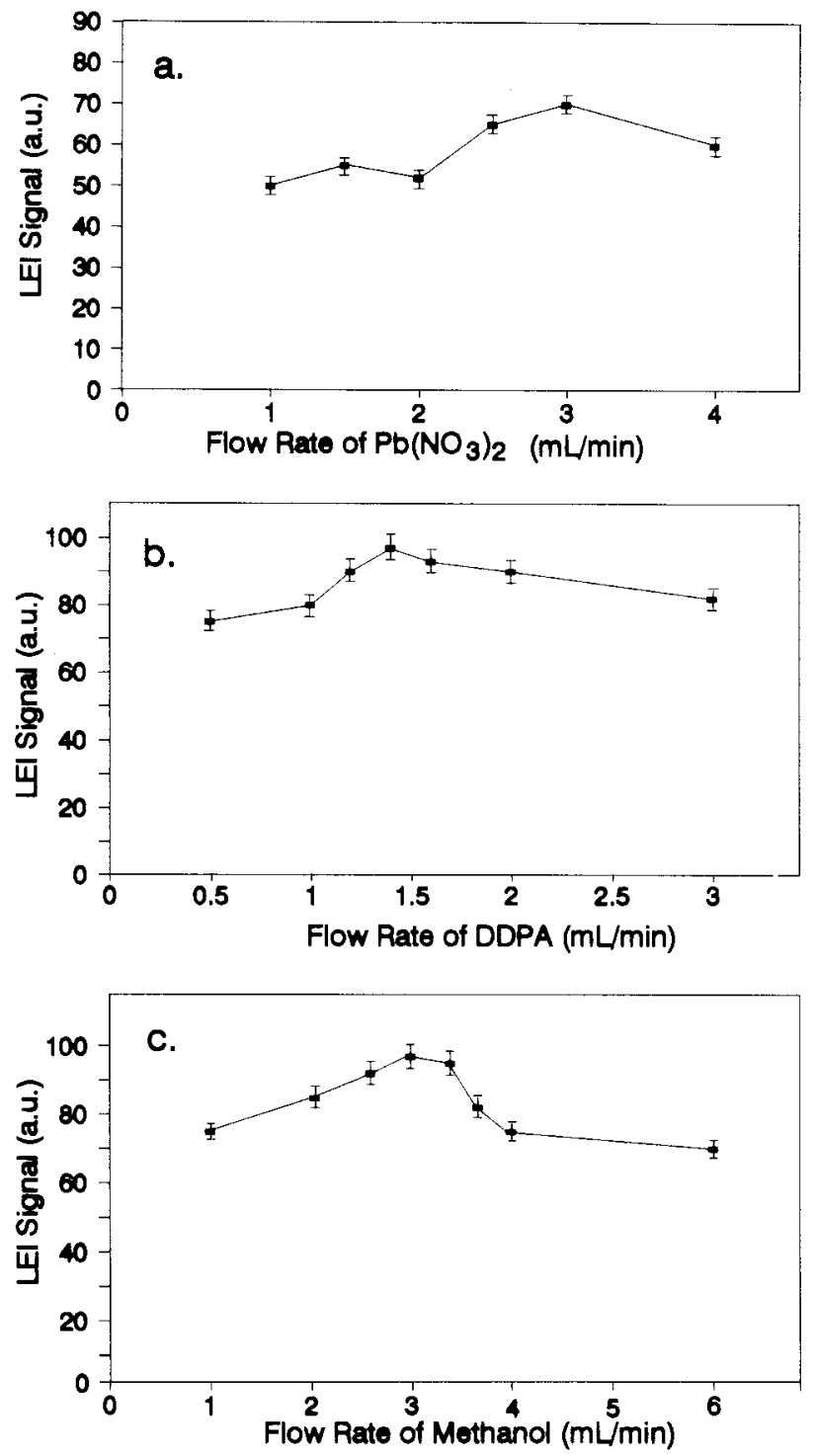

Figure 3. One-step LEI response of $5 \mathrm{~mL}, 1 \mathrm{ppm}$ lead solution to the flow rates of (a) lead sample, (b) DDPA chelating agent at $\mathrm{pH} 1$, and (c) methanol eluent.

with a single laser irradiation at $283.3 \mathrm{~nm}$ hardly detects $\mathrm{Pb}$ at concentrations less than $25 \mathrm{ng} / \mathrm{mL}$ (ppb). Nevertheless, the capabilities of separation and preconcentration allow the FI-LEI spectrometer to achieve enrichment factors of 16- and 48-fold for the volume-fixed 5- and 15-mL sample loading, respectively, in the FI manifold. The enrichment factor is theoretically defined as the ratio of the analyte concentration in the concentrate to that in the original sample. But its value may be practically estimated to be the ratio between the slopes of the calibration curves obtained with and without a preconcentration procedure. ${ }^{22}$ The subsequent limits of $\mathrm{Pb}$ detection, determined as three times the standard deviation $(3 \sigma)$ of the peak height, are evaluated to be 1.48 and $0.51 \mathrm{ppb}$, respectively, for 5 - and $15-\mathrm{mL}$ sample loading.

The sensitivity for lead detection may be improved using a two-step LEI scheme, in which the first laser is fixed at $283.3 \mathrm{~nm}$, while the second laser is tuned to be resonant with the ${ }^{3} P_{1}-{ }^{3} D_{2}$ transition at $600.2 \mathrm{~nm}$. The corresponding laser energies are 50 and $700 \mu \mathrm{J}$, respectively. As shown in Figure 5, the measured signal-to-noise ratio for the LEI peak using direct aspiration is 

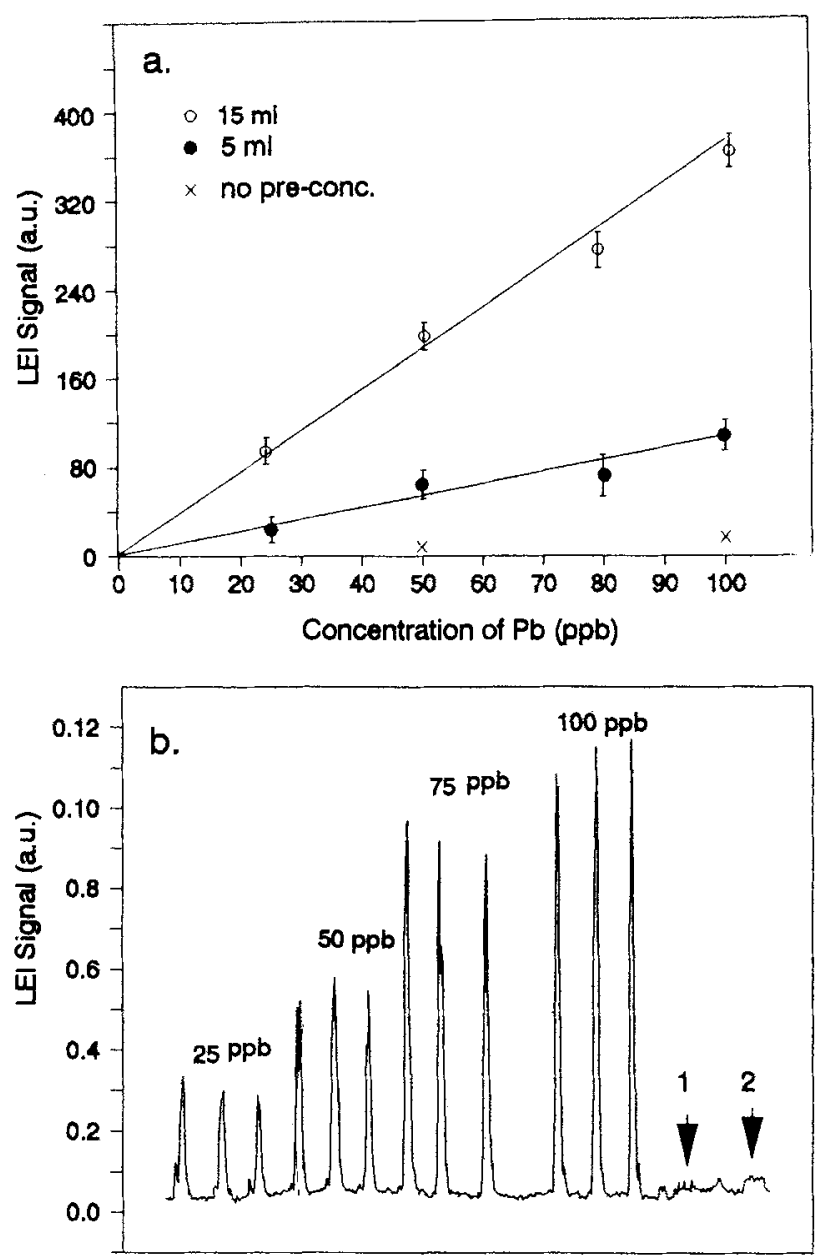

Figure 4. (a) Calibration curves of one-step LEI detection with and without FI coupling; (b) The measured one-step FI-LEI signals for 25, 50, 80, and $100 \mathrm{ppb}$. Arrows 1 and 2 indicate the background noise and one-step LEI intensity of $50 \mathrm{ppb}$ lead solution by direct aspiration, respectively.

greatly enhanced to 160 -fold with respect to the one-step scheme. The ion enhancement obtained is comparable with the value of 210-fold reported by Turk et al. ${ }^{11}$ The lead FI-LEI signal with twostep excitation as a function of sample concentration is measured in Figure 5. Various lead standard solutions are prepared from 250 to $1000 \mathrm{ppt} ; 50 \mathrm{ppm} \mathrm{Na}$ species is added as matrix in each solution. The calibration curve shows a straight line within the concentration range studied. The subsequent detection limits of 0.011 (11 ppt) and $0.0032 \mathrm{ng} / \mathrm{mL}$ (32 ppt) and sampling frequencies of 18 and $9 \mathrm{~h}^{-1}$ for 5 - and $15-\mathrm{mL}$ sample loadings, respectively, are achieved. The results are also listed in Table 1. As expected, the two-step excitation leads to a greater ion yield for detection than the one-step excitation. The ion enhancement is generally attributed to such factors as second-step transition probability, laser intensity, collisional ionization rate coefficients, and transition line width. ${ }^{30,34,35}$ Accordingly, the improved limit of $\mathrm{Pb}$ detection by two-step LEI (Table 1) is a result of at least two factors. First, the deexcitation rate is low, since there are very few lower-lying states in $\mathrm{Pb}$ to facilitate energy transfer. Second, the collisional

(34) Omenetto, N.; Smith, B. W.; Hart, L. P. Fresenius Z. Anal. Chem. 1986 $324,683-697$.

(35) Axner, O.; Rubinsztein-Dunlop, H. Spectrochim. Acta 1989, 44B, 835-866.
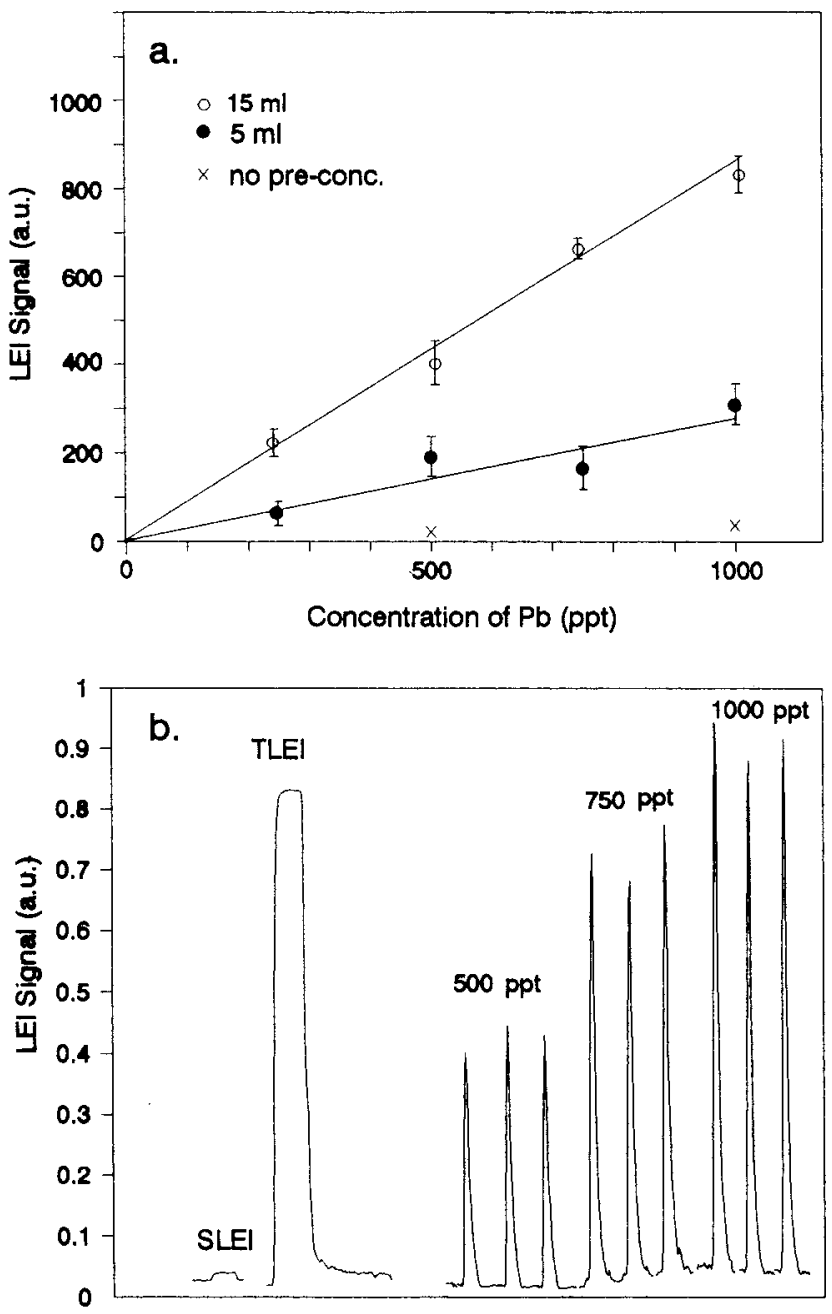

Figure 5. (a) Calibration curves of two-step LEI detection with and without FI coupling. (b) The measured two-step LEI signals for 500, 750 , and $1000 \mathrm{ppb}$. The one-step and two-step LEI signals by direct aspiration are compared.

Table 1. Limit of $\mathrm{Pb}$ Detection and Sampling Frequency for 5- and 15-mL Sample Loading in FI Manifold Using Single-Step and Two-Step LEI Detection

\begin{tabular}{|c|c|c|c|}
\hline \multirow{2}{*}{$\begin{array}{c}\text { aliquot } \\
\text { taken }(\mathrm{mL})\end{array}$} & \multicolumn{2}{|c|}{ detection limit (ppt) } & \multirow{2}{*}{$\begin{array}{c}\text { sampling } \\
\text { frequency }\left(\mathrm{h}^{-1}\right)^{c}\end{array}$} \\
\hline & SLEI & TLEI $^{a}$ & \\
\hline $\begin{array}{r}5 \\
15\end{array}$ & $\begin{aligned} 1480 & \pm 30^{b} \\
510 & \pm 13^{b}\end{aligned}$ & $\begin{array}{r}10.6 \pm 0.3^{b} \\
3.2 \pm 0.1^{b}\end{array}$ & $\begin{array}{r}18 \\
9\end{array}$ \\
\hline
\end{tabular}

a SLEI, single-step LEI; TLEI, two-step LEI. ${ }^{\mathrm{b}}$ One standard deviation was given, evaluated by three replicates. ${ }^{c}$ The number of samples measured per hour.

ionization rate from the state stepwise excited increases with decreasing energy difference with respect to the ionization continuum.

The response sensitivity of FI-LEI in Figures 4 and 5, as evaluated by the slopes of the calibration curves, increases linearly with the loaded volume of the sample solution, regardless of the increase of matrix content with increasing sample volume. This consequence may be a result of two factors. First, LEI detection with water-immersed cylinder electrode exhibits good resistance to the electrical interference induced by the Na matrix. ${ }^{8}$ Second, 


\section{Table 2. Comparison of Detection Limits for $\mathbf{P b}$}

$\begin{array}{lc}\text { trace technique } & \text { detection limit }(\mathrm{ng} / \mathrm{mL}) \\ \text { ETAAS } & 0.0048 \\ \text { FAAS } & 10 \\ \text { GEAAS } & 0.009 \\ \text { spectrophotometer } & 1.5 \\ \text { ETAAS } & 0.0022 \\ \text { FAAS } & 1.7 \\ \text { electrochemistry } & 188 \\ \text { GFAAS } & 0.04 \\ \text { FAAS } & 3 \\ \text { spectrophotometer } & 4.2 \\ \text { spectrophotometer } & 12 \\ \text { electrochemistry } & 200 \\ \text { FAAS } & 4 \\ \text { FAAS } & 10 \\ \text { FAAS } & 2.7 \\ \text { SLEI } & 0.2-0.6 \\ \text { TLEI } & 0.09 \\ \text { FI-SLEI } & 0.51 \\ \text { FI-TLEI } & 0.0032\end{array}$

sample

biological; environmental environmental

lake water; drinking water rice flour

waters

waters

blood

biological; environmental

biological

tap water

port wine

road dust; soil

natural water

waters

waters

waters

waters

seawater

seawater type of separation ref

sorbent extraction 38

sorbent extraction $\quad 39$

ion exchanger 40

ion exchanger 41

sorbent extraction $\quad 29$

sorbent extraction $\quad 42$

none

sorbent extraction $\quad 44$

sorbent extraction $\quad 45$

sorbent extraction $\quad 26$

ion exchanger $\quad 46$

hyride generation $\quad 47$

sorbent extraction $\quad 24$

ion exchanger 48

sorbent extraction $\quad 49$

none 36

none 37

$\begin{array}{ll}\text { sorbent extraction } & b \\ \text { sorbent extraction } & b\end{array}$

38
99
40
47
4
4
4
47
44
49
76
$b$

a ETAAS, electrothermal atomic absorption spectrometer; FAAS, flame atomic absorption spectrometer; GFAAS, graphite furnace atomic absorption spectrometer; SLEI, single-step laser-enhanced ionization by direct aspiration; TLEI, two-step laser-enhanced ionization by direct aspiration. ${ }^{b}$ This work.

the incorporation of a $\mathrm{FI}$ on-line sorbent microcolumn enables effective separation of the lead sample from the interfering matrix. In contrast to the FI-LEI method, the detection of the LEI signal by direct aspiration using two metal rods as collector was found to be suppressed significantly with increasing the Na matrix $>2$ ppm. 5,20

Table 2 shows the comparison of the lead detection limits among various FI-AAS methods and conventional LEI without preconcentration. The flame LEI spectrometer appears to be superior to most AAS methods, especially with the two-step excitation scheme. For instance, Axner and co-workers reported a $\mathrm{Pb}$ detection limit to be $0.2-0.6 \mathrm{ppb}$ using a single laser at the UV wavelength of $280-288 \mathrm{~nm}^{36}$ When a two-step LEI is substituted, the detection limit is lowered to $0.09 \mathrm{ppb}^{37}$ With $\mathrm{FI}$ system, the two-step LEI obtained in this work has further improved the detection limit to $0.0032 \mathrm{ppb}$, which proves to be better than or comparable with those determined by other $\mathrm{FI}$ AAS. ${ }^{38-49}$ Note that the values determined may depend on the loaded volume of the sample.

Matrix Interference. The flame LEI technique has been well developed, but mostly limited to simple aqueous systems. It has

(36) Axner, O.; M agnusson, I.; Petersson, J.; Sjostrom, S. Appl. Spectrosc. 1987, $41,19-26$.

(37) Turk, G. C.; DeVoe, J. R.; Travis, J. C. Anal. Chem. 1982, 54, 643-645.

(38) Yan, X. P.; Adams, F. J. Anal. At. Spectrom. 1997, 12, 459-464.

(39) M a, R.; Adams, F. Spectrochim. Acta 1996, 51B, 1917-1923.

(40) Colognesi, M.; Abollino, O.; Aceto, M.; Sarzanini, C.; M entasti, E. Talanta 1997, 44, 867-875.

(41) Yamane, T.; Yamaguchi, Y. Anal. Chim. Acta 1997, 345, 139-146.

(42) Naghmush, A. M .; Pyrzynska, K.; Trojanowicz, M. Talanta 1995, 42, 851860.

(43) Aldstadt, J .; King, D. F.; Dewald, H. D. Analyst 1994, 119, 1813-1818.

(44) M a, R.; van Mol, W.; Adams, F. Anal. Chim. Acta 1994, 293, 251-260.

(45) Tao, G.; Fang, Z. L. At. A pectrosc. 1996, 17, 22-26.

(46) Lopes, T. I. M. S.; Rangel, O. S. S.; Sartini, R. P.; Zagatto, E. A. G. Analyst 1996, 121, 1047-1050.

(47) Hauser, P. C.; Zhang, Z. P. Fresenius J. Anal. Chem. 1996, 355, 141-143.

(48) Purohit, R.; Devi, S. Anal. Chim. Acta 1992, 259, 53-60.

(49) Lancaster, H. L.; M arshall, G. D.; Gonzalo, E. R.; Ruzicka, J.; Christian, G. D. Analyst 1994, 119, 1459-1465.

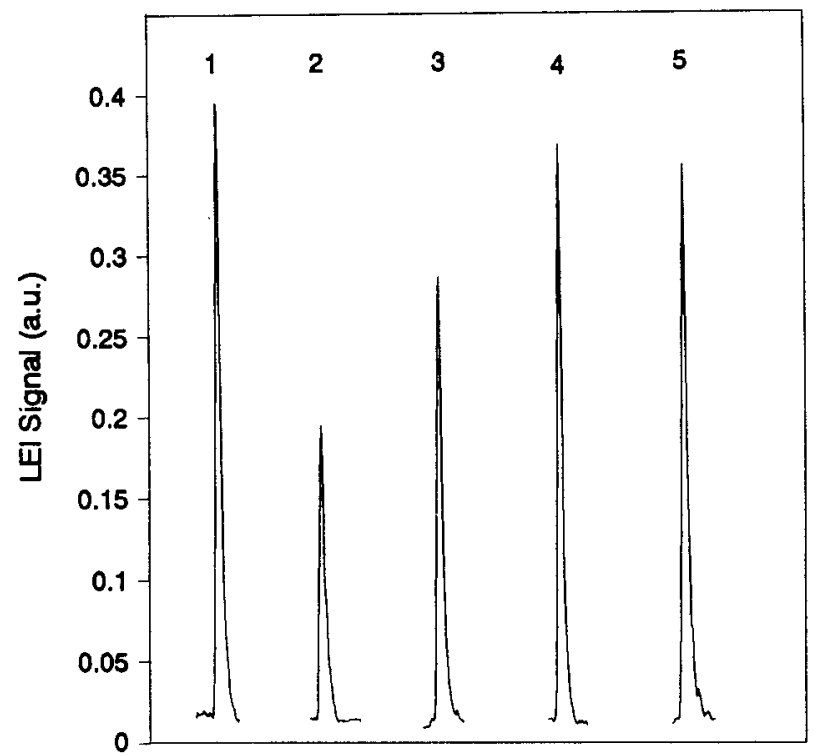

Figure 6. Two-step LEI signals of $5 \mathrm{~mL}, 1 \mathrm{ppm}$ lead solutions with and without $5000 \mathrm{ppm} \mathrm{Na}$ matrix added. LEl signal 1 corresponds to the sample without $\mathrm{Na}$ matrix added, while LEI signals $2-5$ denote the samples each with $5000 \mathrm{ppm}$ Na matrix added. The signals 2-5 are obtained under such conditions that the complex retained in the FI column is rinsed with the deionized water for $0,10,20$, and $30 \mathrm{~s}$ at $6 \mathrm{~mL} / \mathrm{min}$, respectively, before the methanol solvent is eluted.

seldom been applied to real samples associated with the biological or environmental fields, since LEI suffers from the electrical interference induced by those easily ionized matrixes and the flame components. As demonstrated in the last section, the twostep FI-LEI is capable of detecting the aqueous lead solution at the ppt $\left(10^{-3} \mathrm{ng} / \mathrm{mL}\right)$ level, which is about the lead content in the seawater. However, before the technique is applied to the seawater, examination of its capability of resisting against high contents of alkali metal matrixes is crucial. 


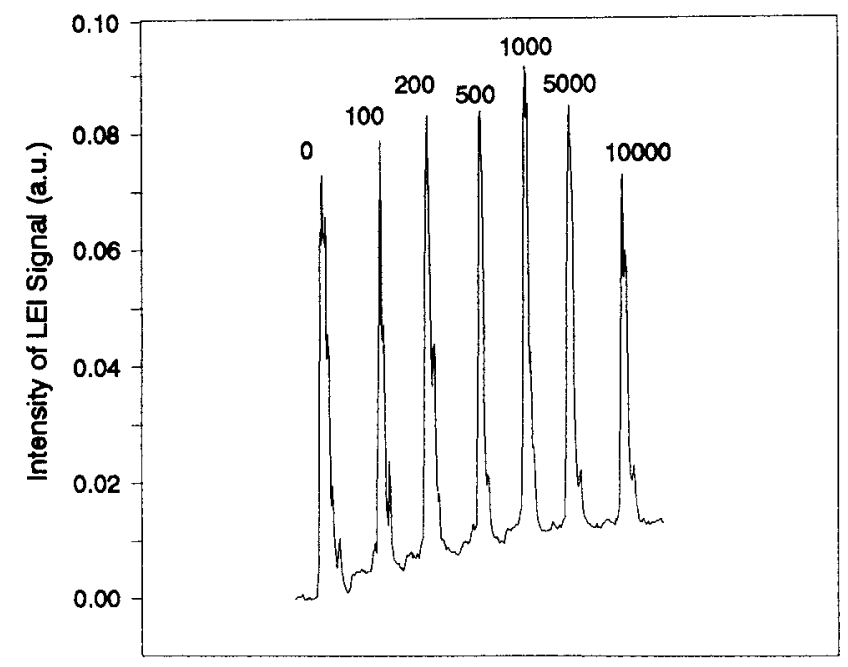

Figure 7. Two-step FI-LEI detection of $5 \mathrm{~mL}, 1 \mathrm{ppm}$ lead solutions containing $0,100,200,500,1000,5000$, and $10000 \mathrm{ppm} \mathrm{Na}$ and $\mathrm{K}$ matrixes, respectively. Deionized water was used to rinse the $\mathrm{Pb}-$ DDPA complex retained in the FI column for $30 \mathrm{~s}$ at $6 \mathrm{~mL} / \mathrm{min}$.

In this study, 100-10000 ppm $\mathrm{K}$ and $\mathrm{Na}$ matrixes were premixed in a $1 \mathrm{ppm} \mathrm{Pb}$ solution, and then $5 \mathrm{~mL}$ of mixed solution was loaded into the FI manifold. The lead sample, forming the complex selectively with the DDPA chelating agent, was retained in the microcolumn, while the alkali matrixes were mostly discarded to the waste. However, as the added matrix increased, a fraction might also be adsorbed. The residual matrix became abundant enough to suppress the resultant lead LEI signal. Figure 6 shows the LEI response to 1 ppm Pb solution containing 5000 ppm Na matrix. The LEI signal is suppressed to $50 \%$ intensity of the original peak obtained without matrix added. Fortunately, the matrix retained in the microcolumn may be significantly removed by rinsing with deionized water. As shown in Figure 6, the LEI signal may be restored up to $90 \%$ intensity, after a rinse with deionized water for $30 \mathrm{~s}$ at a flow rate of $6 \mathrm{~mL} / \mathrm{min}$. Under these optimal conditions, the effect of $\mathrm{Na}$ and $\mathrm{K}$ matrixes was measured in the range of 100-10 $000 \mathrm{ppm}$. Figure 7 indicates that the LEI response maintains almost invariant if the matrix is less than 5000 ppm but reduces to $80 \%$ intensity as the matrix increases to 10000 ppm. As reported, the water-immersed cylinder electrode used in the direct aspiration is able to resist effectively against the $\mathrm{Na}$ matrix up to 3000 ppm. ${ }^{8}$ Beyond that concentration, a severe arcing phenomenon between the collectors may obscure the measurement.

LEI detection as incorporated with a FI on-line sorbent microcolumn proves to be free from the matrix effect. This achievement is of importance to the LEI technique. The electrical interference induced by the matrix has a twofold influence on the $\mathrm{LEI}$ response. First, the interference diminishes the signal-to-noise ratio and causes a weak ion signal to be buried in the background noise. The second effect of the interference may prevent the electric field from reaching the interactive regime, where the sample ions and electrons are generated upon irradiation of a laser. 5,12,13,20 Since the sample ions and electrons cannot be set apart and then collected toward the opposite electrodes by a nonzero electric field, the LEI signal is suppressed and even undetectable.

Detection of $\mathbf{P} \mathbf{b}$ Concentration in Seawater. Taking advantage of the FI capability of separation and preconcentration, the LEI technique was used for the first time to detect trace elements in seawater. With the use of the same conditions optimized for the aqueous $\mathrm{Pb}(\mathrm{II})$ measurement described above, $15 \mathrm{~mL}$ of sample from seawater (NASS-4) was loaded in the FI manifold and then detected with the two-step LEI spectrometer. To remove the residual alkali and alkaline earth matrixes from the microcolumn, the formed $\mathrm{Pb}-\mathrm{DDPA}$ complex was rinsed with deionized water for $30 \mathrm{~s}$ at $6 \mathrm{~mL} / \mathrm{min}$. The quantity of $\mathrm{Pb}$ content was determined by using a standard addition method, yielding a result of $0.0112 \pm 0.0006 \mathrm{ppb}$. The results for $\mathrm{Pb}$ in seawater are excellent, consistent with the certified value of $0.013 \pm 0.005 \mathrm{ppb}$.

\section{CONCLUSION}

In this work, the LEI technique coupled with the FI system is used to detect trace lead amounts in aqueous solution and seawater. The capability of $\mathrm{FI}$ separation and preconcentration enables the two-step LEI to achieve a detection limit of $3.2 \mathrm{ppt}$ from the aqueous lead solution at a sampling frequency of $9 \mathrm{~h}^{-1}$. The enrichment factor reaches 16- and 48-fold for the 5- and 15$\mathrm{mL}$ sample loading, respectively. The LEI and FI coupling also increases the tolerance of matrix interference by a factor of $\sim 4$. $\mathrm{LEI}$ detection is finally applied to the analysis of $\mathrm{Pb}$ content in seawater, achieving a result consistent with the certified value.

\section{ACKNOWLEDGMENT}

The authors thank L. S. Huang for his help in the experiments. This work is supported by National Science Council of Republic of China under Contract NSC88-2113-M -002-010.

Received for review August 28, 1998. Accepted January 20, 1999.

AC980970T 